Formación Universitaria

Vol. 7(5), 15-22 (2014)

doi: $10.4067 /$ S0718-50062014000500003

\title{
Enseñanza del Concepto de Fuerzas Intermoleculares en Ingeniería Química: su Relación con Propiedades Medibles
}

\author{
Orlando J. Domínguez, María A. Toro, Emilio M. Serrano \\ Facultad de Ingeniería, Universidad Nacional de Salta. Consejo de Investigación de la Universidad Nacional \\ de Salta. Av. Bolivia 5150, 4400 - Salta, Argentina (email: orlando@unsa.edu.ar, eserrano@unsa.edu.ar)
}

Recibido May. 30, 2014; Aceptado Jul. 14, 2014; Versión final recibida Sep. 10, 2014

\begin{abstract}
Resumen
Para favorecer la compresión del concepto fuerzas intermoleculares, que presenta dificultades en su conceptualización como consecuencia de requerir el conocimiento de procesos a nivel molecular. Para ello, se plantea realizar tareas experimentales que posibilitan establecer relaciones entre los tipos de fuerzas involucradas con la estructura y las propiedades macroscópicas. La experiencia en laboratorio consiste en medir una propiedad macroscópica como la tensión superficial, para diferentes sustancias y relacionar estas medidas con las fuerzas de interacción y su naturaleza. Se evaluó el conocimiento adquirido utilizando el laboratorio didáctico propuesto, comparando con evaluaciones donde se había aplicado enseñanza teórica clásica. El laboratorio propuesto produjo notables mejoras con respecto a la forma clásica de enseñanza.
\end{abstract}

Palabras clave: fuerzas intermoleculares, tensión superficial, laboratorio didáctico, enseñanza e la ingeniería

\section{The Teaching of the Concept of Intermolecular Forces in Chemical Engineering: their Relation to Measurable Properties}

\begin{abstract}
To facilitate the understanding of the concept of intermolecular forces which presents several difficulties as it requires the comprehension of processes occurring at molecular level. To do this, a lab experiment that enables to establish relationships between the different types of forces involved with the structure and macroscopic properties is proposed. Laboratory experiments are designed to measure a macroscopic property such as the surface tension for different substances, and relating these measurements with the interaction forces and their nature. Evaluation of the results using the knowledge acquired with the proposed didactic laboratory is done and results compared with classical theoretical learning. The proposed lab showed important improvements with respects to classical teaching.
\end{abstract}

Keywords: intermolecular forces, surface tension, didactic laboratory, engineering teaching 


\section{INTRODUCCIÓN}

A partir del año 2005, la carrera de Ingeniería Química que brinda la Facultad de Ingeniería de la Universidad de Salta (Salta, Argentina), ha cambiado su plan de estudio, pasando de 6 a 5 años de cursado. Este proceso de cambio requiere, según Domínguez y Michel (2010), el abordaje de nuevas estrategias y metodologías en la enseñanza. En la carrera de Ingeniería Química, y especialmente en la enseñanza de la química general, para alumnos del primer año del nivel universitario, es fundamental la búsqueda de nuevas estrategias para solucionar algunos problemas de aprendizaje. Sobre todo de aquellos conceptos difíciles de comprender que requieren altos niveles de abstracción, como es el caso del estudio de las fuerzas intermoleculares.

La comprensión de esos conceptos en Ingeniería Química es un reto, debido justamente a su naturaleza teórica y a la dificultad para ponerlos en práctica. Dicha comprensión implica un triple requisito: por una parte, requiere la comprensión del concepto a nivel macroscópico, lo cual implica poder aplicar el concepto a distintos casos. El segundo requisito, conlleva entender su significado a nivel microscópico, como proceso que ocurre a nivel atómico-molecular. Finalmente, exige entender las relaciones establecidas entre el nivel microscópico y el macroscópico a través del uso de representaciones simbólicas, esquemas, fórmulas y ecuaciones químicas (Solsona et al., 2003).

Es así que antes de llevarse a cabo la concreción de las ideas a nivel de aula, las mismas deben surgir de las investigaciones en Didáctica de las Ciencias Naturales, las que requieren poder transformar los conocimientos construidos previamente en el campo de la investigación científica en propuesta de aplicación práctica (Bucat, 2004), lo que constituye un verdadero desafío para los docentes de química (Gil y Pessoa, 2000). Por ello, a pesar que se han hecho numerosos intentos por hacer explícita la naturaleza de la química (Lorenzo, 2012, Weininger, 1998) y los beneficios de su incorporación al currículum, es un aspecto pendiente en la enseñanza de la química (Lombardi y Labarca, 2007). Un posible camino para que los docentes comprendan la naturaleza de la química, y puedan posteriormente enseñarla, es tomar como base el ya clásico modelo de Alex Johnstone $(1982,1993,2000)$ de gran utilidad desde el punto de vista didáctico que ha sido ampliamente discutido y reinterpretado por los autores (Gilbert y Treagust, 2009, Talanquer, 2011).

Alex Johnstone (1982), propone relacionar los tres vértices del triángulo, en un vértice ubica el conocimiento por la observación macroscópico que representa un nivel descriptivo; el otro vértice lo constituyen el conocimiento representado por el nivel sub-microscópico, explicativo y el tercer vértice estaría expresado por un nivel representativo dado por una estructura. En este trabajo se pretende explicar estas interrelaciones entre tensión superficial, su estructura y fuerzas intermoleculares. Mientras que Labarca (2013) en su obra "Otras miradas de la ciencia" expresa que estas interrelaciones son distintas representaciones del mismo conocimiento.

El aprendizaje de las fuerzas intermoleculares presenta dificultades en su conceptualización como consecuencia de requerir el conocimiento de procesos a nivel molecular. Se hace necesario recurrir a conceptos más concretos, implementando tareas experimentales o bien laboratorios didácticos, a partir de las cuales es posible realizar abstracciones que posibiliten establecer relaciones significativas entre los tipos de fuerzas involucradas y su relación con las estructuras y las propiedades macroscópicas que se ven afectadas (puntos de ebullición y de fusión, calor de vaporización). La experiencia en laboratorio, consiste en la medición de la tensión superficial para cada una de las sustancias en estudio, por lo que a continuación se define esta propiedad y posteriormente se da los fundamentos para su determinación experimental.

Termodinámicamente la tensión superficial es un fenómeno de superficie y es la tendencia de un líquido a disminuir su superficie hasta que su energía de superficie potencial sea mínima, condición necesaria para que el equilibrio sea estable. Como la esfera presenta un área mínima para un volumen dado, entonces por la acción de la tensión superficial, la tendencia de una porción de un líquido lleva a formar una esfera o a que se produzca una superficie curva o menisco cuando un líquido entra en contacto con un recipiente. Como resultado de la existencia de la tensión superficial, es necesario entregar energía a un sistema para aumentar su superficie. La tensión superficial $\gamma$ se puede interpretar, entonces, como la energía que se debe dar a un sistema líquido para aumentar su superficie en una unidad, venciendo la atracción entre sus moléculas (Castellan, 1987; Atkins, 2006).

$\gamma=\Delta \mathrm{E} / \Delta \mathrm{A}$

En donde la energía suministrada $(\Delta \mathrm{E})$ es un escalar, la tensión superficial $\gamma$ es un vector que tiene una dirección tangencial a la superficie antes de ampliar el área en un $\Delta \mathrm{A}$. 
Uno de los factores que afectan al valor de la tensión superficial es la intensidad de las fuerzas intermoleculares, de modo que un mayor valor de $\gamma$ implica mayor fuerza de atracción intermolecular a vencer para aumentar su superficie en una unidad. En las figuras 1a y $1 \mathrm{~b}$ se observan un alambre doblado en forma de $U$, que con un alambre vertical móvil forma un rectángulo (bastidor) que encierra una película líquida. La figura $1 \mathrm{~b}$, presenta el alambre vertical desplazado a la derecha, ocasionando un incremento en $\mathrm{dx}$ con respecto del sistema o de la película original. La película extendida en $\mathrm{dx}$ realiza trabajo, para aumentar el área.

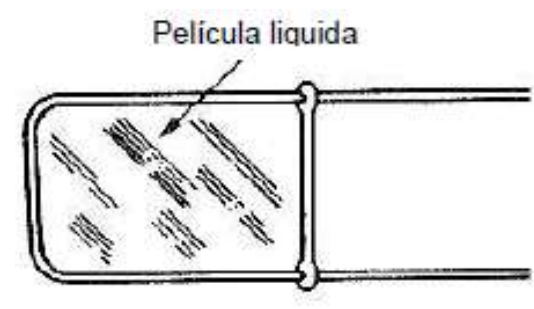

Fig. 1a: Bastidor con líquido, película original.

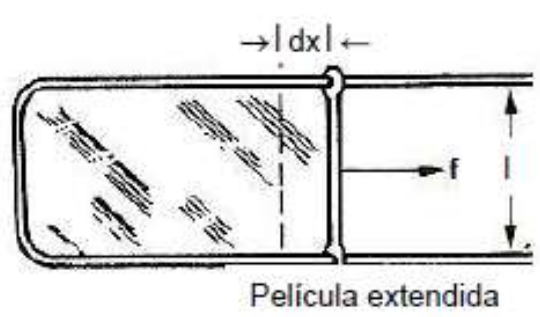

Fig. 1b: Bastidor, película extendida en dx.

De acuerdo a la definición general de trabajo, corresponde al producto de la fuerza f actuante en el sentido del desplazamiento multiplicado por el desplazamiento $\mathrm{dx}$ provocado:

$W=f . d x$

Pero: $f=\gamma \cdot(2 \mathrm{I})$, ya que la película de líquido se forma en ambas caras del bastidor de alambre. De modo que la expresión (2) queda expresada de la siguiente manera:

$W=\gamma \cdot(2 I) \cdot d x=\gamma \cdot d A$

Siendo dA el cambio de la superficie del líquido, provocado por el desplazamiento.

\section{MÉTODOLOGÍA}

Tradicionalmente, el aprendizaje del tópico "fuerzas intermoleculares" se realiza de manera teórica, basado en un razonamiento abstracto, sin apelar a fenómenos observables y medibles que justifiquen el fenómeno en estudio. Por ello, se propone consolidar el concepto de fuerzas intermoleculares a partir de la obtención de valores experimentales de la tensión superficial realizada con la ayuda de un equipo de medición, tensiómetro que lleva el nombre en honor a Pierre Lecomte Du Noüy reconocido por sus trabajos en tensión superficial. El equipo utilizado para realizar las tareas experimentales, es el que muestra la figura $2 a$, en tanto en la figura $2 \mathrm{~b}$ se presenta en detalle del anillo con la película, sobre el que ejerce la tensión superficial.

Para comenzar la determinación de la tensión superficial $(\gamma)$, se coloca el líquido en el platillo. Se enraza el anillo con la superficie del líquido en el platillo a través del tornillo de nivelación. Con el dial de torsión se comienza a trasmitir una fuerza f ejercida por la torsión del alambre, transmitiéndose al líquido a través de un anillo de radio $R$, siendo el lado del bastidor $I=(2 \pi R)$ y el anillo tiene un cierto espesor que es el del alambre (2r), restituyendo la no deformación de la superficie lisa del líquido al que se quiere determinar su tensión superficial $(\gamma)$, mediante el tornillo inferior (figura 2.a). Para las experiencias en laboratorio se utilizó un tensiómetro de Du Noüy, marca KRUSS, modelo RD 5367/62. Para realizar la medición colocar el líquido en el platillo, hasta que el anillo quede rasante a la superficie del mismo. Accionar la manija de la escala que genera la torsión del alambre y nivelar la posición del líquido siempre rasante al anillo. Repetir esta operación hasta que se rompa el contacto del anillo con el líquido. Reproducir la experiencia para verificar la medición.

Como el anillo tiene dos caras aparece un 2 en la ecuación:

$f=2(2 \pi R) \gamma$

En la ecuación (4) debe colocarse un factor $k$ de corrección que depende del radio $\mathrm{R}$ del anillo y espesor $2 \mathrm{R}$ del alambre del anillo, que en nuestra experiencia se determinará experimentalmente:

$f=2(2 \pi R) \gamma \cdot k$ 


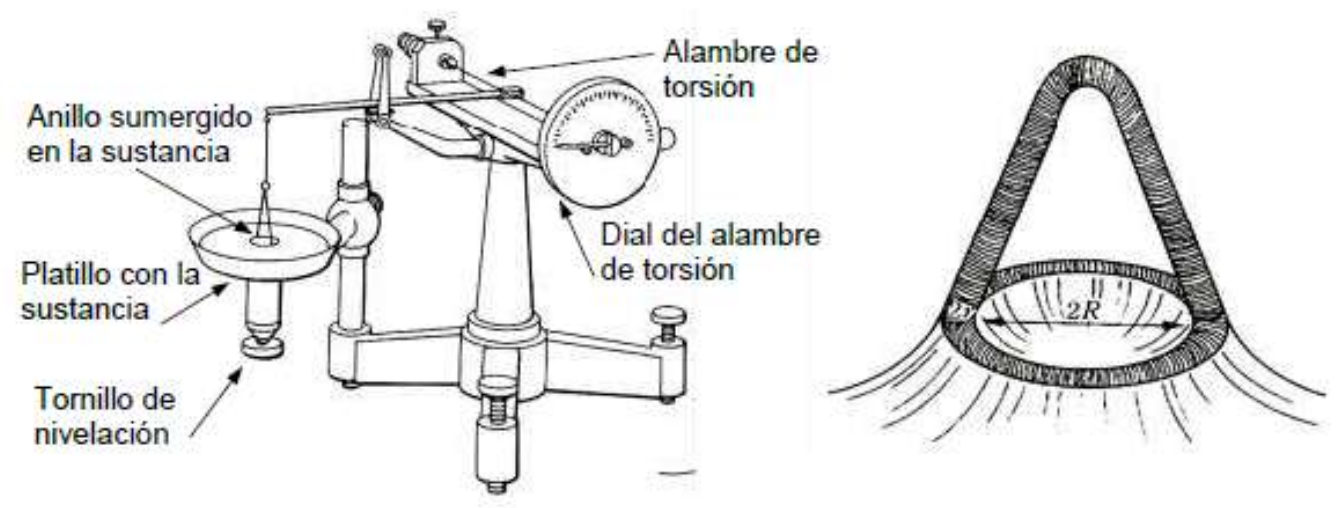

Fig. 2a: Equipo tensiómetro de Du Noüy

Fig. 2b: Anillo del tensiómetro de Du Noüy

Para determinar el factor $\mathrm{k}$ del equipo, se realiza la calibración con agua destilada en el platillo, obteniendo un valor de $\mathrm{k}=(72,8 / \mathrm{L})$, donde $\mathrm{L}$ es la lectura de la rotura del contacto usando agua. Multiplicar todas las lecturas por el factor determinado.

Trabajar con las siguientes sustancias para efectuar una discusión sobre los resultados y relacionar con la intensidad de las fuerzas intermoleculares y con otras propiedades especiales, como benceno, n-hexano, alcohol etílico, 1-propanol, etilenglicol, glicerina, agua y mercurio. Las mediciones se deben realizar a temperatura ambiente $\left(25^{\circ}\right)$. Finalmente, los alumnos realizan las mediciones, determinando los valores leyendo y registrando los mismos en una tabla.

\section{RESULTADOS Y DISCUSION}

Los valores tabulados para cada una de las sustancias puras utilizadas, se presenta en la tabla 1 , se obtuvieron de un software comercial (Component Plus, 2005). Se observa que los valores de la tensión superficial $(\gamma)$, entre los valores leídos con los extraídos del software comercial (Component Plus, 2005), presentan una diferencia que va entre $0,6 \%$ a $7,1 \%$, que son valores aceptables de error entre valores teóricos y experimentales. Para efectuar el análisis es necesario conocer la geometría molecular de cada compuesto y contar con los valores de las propiedades afectadas por el tipo de intensidad de las fuerzas intermoleculares.

Tabla 1: Valores de tensión superficial, tabulados y experimentales.

\begin{tabular}{|c|c|c|c|c|c|c|c|c|}
\hline & $\mathrm{C}_{6} \mathrm{H}_{6}$ & $\mathrm{C}_{6} \mathrm{H}_{14}$ & $\mathrm{C}_{2} \mathrm{H}_{5}(\mathrm{OH})$ & $\mathrm{C}_{3} \mathrm{H}_{7}(\mathrm{OH})$ & $\mathrm{C}_{2} \mathrm{H}_{4}(\mathrm{OH})_{2}$ & $\mathrm{C}_{3} \mathrm{H}_{5}(\mathrm{OH})_{3}$ & $\mathrm{H}_{2} \mathrm{O}$ & $\mathrm{Hg}$ \\
\hline $\begin{array}{c}\gamma \text { [dina/cm] } \\
\text { Tabulado }\end{array}$ & 28,2 & 17,9 & 22,1 & 23,41 & 47,9 & 63,1 & 72,8 & 472 \\
\hline $\begin{array}{c}\gamma[\text { dina/cm] } \\
\text { Experimental }\end{array}$ & 30,2 & 18,9 & 23,2 & 24,9 & 48,2 & 60,5 & 70,5 & $\begin{array}{l}\text { Fuera de } \\
\text { escala }\end{array}$ \\
\hline
\end{tabular}

El problema a resolver es una vez determinados los valores de tensión superficial de diferentes sustancias a través de la experiencia, inferir su dependencia con las fuerzas intermoleculares atractivas y buscar su interpretación en función de sus estructuras y los grupos polares presentes en las distintas sustancias. Por otro lado, se compararon los resultados del aprendizaje logrado a través de esta experiencia de laboratorio versus la enseñanza tradicional teórica. Se tomó un periodo de análisis de 4 años en total, comparando los dos primeros usando la metodología clásica teórica frente a los dos últimos años aplicando la metodología de enseñanza por medio de tareas experimentales. Esta comparación arrojó un incremento del $20 \%$ de aprobados en las evaluaciones por tema, evaluación que se realiza una vez finalizado el trabajo experimental. Además, se observó un incremento de $18 \%$ en la cantidad de alumnos que aprobaron la evaluación parcial donde se involucraba el tema en cuestión.

Para aumentar el área de un líquido es necesario entregar energía al sistema, y esa energía por unidad de área generada es igual a la tensión superficial, y siendo necesario vencer las fuerzas de atracción intermolecular para generar esa nueva superficie. Por lo que un aumento en el valor de la tensión superficial es indicativo de la intensidad de las fuerzas de atracción que actúan entre las moléculas del sistema líquido. 
Tabla 2: Valores de propiedades relacionados con las fuerzas intermoleculares (Component Plus Program, Pure Components Properties, 2005).

\begin{tabular}{|c|c|c|c|c|c|c|c|c|}
\hline Propiedad & $\begin{array}{c}\text { Benceno } \\
\mathrm{C}_{6} \mathrm{H}_{6}\end{array}$ & $\begin{array}{l}\text { Hexano } \\
\mathrm{C}_{6} \mathrm{H}_{14}\end{array}$ & $\begin{array}{c}\text { Etanol } \\
\mathrm{C}_{2} \mathrm{H}_{5}(\mathrm{OH})\end{array}$ & $\begin{array}{l}\text { 1-Propanol } \\
\mathrm{C}_{3} \mathrm{H}_{7}(\mathrm{OH})\end{array}$ & $\begin{array}{r}\text { Etanodiol } \\
\mathrm{C}_{2} \mathrm{H}_{4}(\mathrm{OH})_{2}\end{array}$ & $\begin{array}{r}\text { Propanotriol } \\
\mathrm{C}_{3} \mathrm{H}_{5}(\mathrm{OH})_{3}\end{array}$ & $\begin{array}{c}\text { Agua } \\
\mathrm{H}_{2} \mathrm{O}\end{array}$ & $\begin{array}{c}\text { Mercurio } \\
\mathrm{Hg}\end{array}$ \\
\hline Estructura & & & $\mathrm{I}_{\mathrm{OH}}$ & & I & & & $\mathrm{Hg}$ \\
\hline $\begin{array}{l}\text { momento } \\
\text { dipolar [D] }\end{array}$ & 0,000 & 0,000 & 1,69 & 1,68 & 2,31 & 4,197 & 1,849 & -- \\
\hline $\begin{array}{c}\gamma[\mathrm{dina} / \mathrm{cm}] \\
\text { Tabulado }\end{array}$ & 28,2 & 17,9 & 22,1 & 23,41 & 47,9 & 63,1 & 72,8 & 472 \\
\hline $\begin{array}{c}\gamma[\mathrm{dina} / \mathrm{cm}] \\
\text { Experimental }\end{array}$ & 30,2 & 18,9 & 23,2 & 24,9 & 48,2 & 60,5 & 70,5 & \begin{tabular}{|l|} 
Fuera \\
de \\
escala
\end{tabular} \\
\hline $\begin{array}{l}\mathrm{Pv} 25^{\circ} \mathrm{C} \\
{[\mathrm{mm} \mathrm{Hg}]}\end{array}$ & 140,9 & 151 & 58,9 & 20,46 & $3,6 \times 10^{-4}$ & $1,6 \times 10^{-4}$ & 23,57 & $\begin{array}{c}1,93 x \\
10^{-3} \\
\end{array}$ \\
\hline $\mathrm{T}^{\circ} \mathrm{eb}\left[{ }^{\circ} \mathrm{C}\right]$ & 60,2 & 68,9 & 78,4 & 97,21 & 197 & 288 & 100 & 356,7 \\
\hline $\begin{array}{l}\text { Fuerzas } \\
\text { Intermole- } \\
\text { culares }\end{array}$ & London & London & $\begin{array}{l}\text { Enlaces } \\
\text { hidrógeno }\end{array}$ & $\begin{array}{l}\text { Enlaces } \\
\text { hidrógeno }\end{array}$ & $\begin{array}{l}\text { Enlaces } \\
\text { hidrógeno }\end{array}$ & $\begin{array}{l}\text { Enlaces } \\
\text { hidrógeno }\end{array}$ & $\begin{array}{c}\text { Enlaces } \\
\text { hidrógeno }\end{array}$ & $\begin{array}{l}\text { Unión } \\
\text { metá- } \\
\text { lica }\end{array}$ \\
\hline $\begin{array}{c}\text { Calor de } \\
\text { Vaporización } \\
\text { (KJ/mol) }\end{array}$ & 31,94 & 28,78 & 38,65 & 41,65 & 52,78 & 66,39 & 40,81 & 58,96 \\
\hline
\end{tabular}

A través de estas experiencias de laboratorio se puede entonces inferir en la enseñanza de la relación entre lo medido (tensión superficial) y lo estudiado (fuerzas intermoleculares). Para ello, se analizan los valores de las propiedades relacionadas a las fuerzas intermoleculares agrupándolas de acuerdo a la similitud estructural que presentan, como pueden observarse en la tabla 2. Alcoholes con el mismo número de átomos de carbono pero con distintas cantidades de grupos funcionales $-\mathrm{OH}$. Es el caso de los pares etanol/etanodiol y propanol/propanotriol.

Se observa que la tensión superficial aumenta notoriamente al aumentar el número de grupos $-\mathrm{OH}$, por ejemplo, $\gamma_{\text {etanol }}=2,32 \cdot 10^{-4} \mathrm{~N} / \mathrm{m}$ y $\gamma_{\text {etanodiol }}=4,82.10^{-4} \mathrm{~N} / \mathrm{m}$; es decir, que las fuerzas intermoleculares son más intensas cuanto mayor es el número de grupos $-\mathrm{OH}$. Por lo que a mayor número de grupos funcionales $\mathrm{OH}$ aumenta el número de uniones polares en la estructura, aumentando los puntos de atracción entre las moléculas. También es de esperar que se produzca en el mismo sentido, un aumento en los valores de calor de vaporización y temperaturas normales de ebullición. Esto queda corroborado con los datos presentados en la tabla 2.

Moléculas con igual número de átomos de carbono pero distintos arreglos espaciales, como benceno y nhexano: El valor de $\gamma$ correspondiente al benceno es mayor que el del hexano. Esto sólo se podría atribuir a la diferencia en sus estructuras espaciales ya que dependiendo de los arreglos moleculares existirán más o menos puntos de contacto entre las moléculas. En el caso del benceno su estructura planar permite mayor interacción atractiva que en el caso del n-hexano, de estructura en zigzag, aun cuando ambas estructuras son apolares (momento dipolar cero).

Alcoholes con distintos número de átomos de carbono e igual número de grupos funcionales -OH; es el caso del par etanol/propanol: Prácticamente los valores experimentales de tensión superficial son similares para ambos compuestos, no hay diferencias significativas, lo cual nos indica que la fortaleza de las fuerzas de atracción son de la misma magnitud y centradas en la existencia de un único grupo $-\mathrm{OH}$. Se esperaría que las otras propiedades tales como presión de vapor, temperatura de ebullición y calor de vaporización sean también del mismo orden. Sin embargo las presiones de vapor son marcadamente diferentes (mayor para el propanol) y esto puede atribuirse al tamaño molecular, pero no se ve afectada la intensidad de la interacción molecular. 
Si se compara el agua con los otros líquidos, su tensión superficial resulta ser la mayor (excepto comparada con el valor del mercurio), por lo que se esperaría mayores valores en el momento dipolar, el calor de vaporización y la temperatura de ebullición para el agua. Esta predicción se verifica sólo cuando se compara las propiedades del agua con las del etanol y del propanol, que tienen en su estructura un solo grupo -OH. Pero cuando se contrastan las mismas propiedades con los valores correspondientes a los polioles de (etilenglicol y glicerina), esto no ocurre así ya que éstos últimos tienen los valores de las propiedades mayores que para el agua, aun teniendo menores valores de tensión superficial. Esto se explica si se tiene en cuenta sus estructuras con dos y tres grupos $-\mathrm{OH}$, lo cual ayuda a establecer mayor cantidad de enlaces unión hidrógeno por molécula que en el caso del agua. Sustancias con estructuras moleculares y la unión metálica $(\mathrm{Hg})$ : Por su alto valor permite comparar la fuerte unión que hay entre los núcleos de los metales debido al "mar de electrones" (unión metálica).

En la tabla 2, se presentan otras propiedades microscópicas como el momento dipolar, expresado en Debye que es una cuantificación de la polaridad molecular, al igual que las propiedades intensivas macroscópicas como presión de vapor (Pv) a temperatura estándar, temperatura normal de ebullición (Toeb) y el calor latente de vaporización a los fines de relacionarlos con el estudio de las fuerzas intermoleculares. Esta relación entre propiedades hace posible profundizar los puentes conceptuales, entre la diversidad de escalas y dimensiones en las que tales modelos se definen, según lo expresa Talanquer (2009), para con ello afianzar los conocimientos adquiridos. Los valores de la tensión superficial no se expresaron en el SI por ser los valores a experimentar que se leen directamente en dina $/ \mathrm{cm}$.

\section{CONCLUSIONES}

La importancia que implica esta práctica en una materia de química general, es que desarrolla un conocimiento significativo de fuerzas intermoleculares y podría ayudar a mejorar la comprensión de temas posteriores como las desviaciones de la ley de Raoult, propiedades coligativas y de todas las funciones en exceso de mezclas.

Se puede relacionar el trabajo del profesor Alex Johnstone (1982) con él presentado aquí, en donde los tres vértices del triángulo, son representados. Ubicando en un vértice el conocimiento macroscópico (nivel descriptivo), representado por las determinaciones medibles de tensión superficial; el otro vértice lo constituyen las fuerzas intermoleculares, que forman parte de la micro química (nivel explicativo), y el tercer vértice está descrito por un modelo geométrico molecular (nivel representativo). Siendo estas tres representaciones complementos que permiten profundizar el conocimiento del tema.

En el proceso de enseñanza como en el de aprendizaje, las visiones de los estudiantes sobre la metodología de obtención e interpretación de datos y determinación de valores, compiten con nociones y conceptos que el profesor planifica desarrollar mediante clases teóricas magistrales. Estos procesos se ven favorecidos por un sistema participativo como lo son los laboratorios experimentales.

Aplicada esta metodología, los alumnos son capaces de construir un conocimiento más sólido y profundo que aquellos otros alumnos de años anteriores que aprendieron sobre la base de la metodología tradicional. En este sentido se observó un incremento en el rendimiento académico en los alumnos que aprobaron la evaluación del tema, al igual que el número de alumnos que aprobaron la evaluación parcial que involucra el tema en cuestión.

Explorando las múltiples relaciones entre los diferentes elementos que entran en juego cuando el profesor y los estudiantes realizan un trabajo de laboratorio, muchos otros temas emergerán, posibilitando una profundización en el estudio de esta modalidad de enseñanza. Por lo que el estudio realizado no se agota con lo presentado hasta aquí, ya que es posible utilizar otros aspectos que permitan enriquecer aún más el conocimiento del tema y ahondar su estudio.

\section{REFERENCIAS}

Atkins, Peter y Jones Loretta, Principios de Química. 3ra edición. Ed. Médica Panamericana., 161-171Buenos Aires, Argentina. (2006).

Bucat, R., Pedagogical content knowledge as a way forward: applied research in chemistry education, Chemistry Education: Research and Practice, 5 (2), 215-228. (2004).

Castellan, Gilbert W., Fisicoquímica. 2da Edición. Editorial Addison - Wesley Iberoamericana. 434-436. Wilmington, Delaware. USA. (1987). 
Component Plus Program, Pure Components Properties. ProSim. Labège, Toulouse. Francia. (2005).

Domínguez, Orlando J. y Raquel L. Michel. Mejoras en el Desarrollo del Proyecto Final para Estudiantes de Ingeniería Química. Formación Universitaria. Vol. 3(4), 47-52 (2010)

Gil, D. y Pessoa, A.M., Dificultades para la incorporación a la enseñanza de los hallazgos de la investigación e innovación en didáctica de las ciencias, Educación Química, 11(2), 250-257 (2000).

Gilbert, J. K., and D. F. Treagust. Towards a Coherent Model for Macro, Submicro and Symbolic Representations in Chemical Education. In Multiple Representations in Chemical Education, ed. John Gilbert, David Treagust, 333-358. The Netherlands: Springer. (2009).

Johnstone, A. Macro- and micro-chemistry. School Science Review, vol. 64, 377-379. (1982).

Johnstone, A., The development of Chemistry teaching. Journal of Chemical Education, 70 (9), 701-705 (1993).

Johnstone, A. H., Teaching of chemistry - logical or psychological? Chemistry Education: Research and Practice in Europe, 1 (1), 9-15 (2000).

Labarca, Martín. Otras miradas de la Ciencia. Educación en la Química. Conferencia, Actas de las XVI reunión de Educadores en la Química - REQ XVI - ADEQRA. 1-18. Bahía Blanca, Buenos Aires, Argentina. (2013).

Lombardi, O. y M. Labarca, The philosophy of chemistry as a new resource for chemistry education. Journal of Chemical Education, 84 (1), 187-192. (2007).

Lorenzo, María Gabriela, Los formadores de profesores: el desafío de enseñar enseñando. Teacher's educators: The challenge of teaching trough teaching. Revista de Curriculum y formación de profesorado. Vol(16), № 2 (2012). http://www.ugr.es/local/recfpro/rev162COL3.pdf [último acceso: 09/05/14]

Solsona, N., Izquierdo, M., y De Jong, O., Exploring the development of students conceptual profiles of chemical change. International Journal of Science Education, 25(1), 3-12. (2003)

Talanquer, V., Construyendo puentes conceptuales entre las varias escalas y dimensiones de los modelos químicos. Rev. Educación Química - EduQ Numero 5. 11-17. (2009).

Talanquer, V., Macro, submicro and symbolic: The many faces of the chemistry "triplet". International Journal of Science Education, 33 (2), 179-195. (2011).

http://dx.doi.org/10.1080/09500690903386435 [último acceso 04/03/14]

Weininger, S. J., Contemplating the finger: Visuality and the semiotics of chemistry. HYLE, International Journal for Philosophy of Chemistry, 4 (1), 3-27. (1998). 
